\title{
Performance comparison of the MOXY and PortaMon near-infrared spectroscopy muscle oximeters at rest and during exercise
}

Chris J. McManus

Jay Collison

Chris E. Cooper 


\title{
Performance comparison of the MOXY and PortaMon near-infrared spectroscopy muscle oximeters at rest and during exercise
}

\author{
Chris J. McManus, * Jay Collison, and Chris E. Cooper \\ University of Essex, School of Sport, Rehabilitation and Exercise Sciences, Colchester, United Kingdom
}

\begin{abstract}
The purpose of the study was to compare muscle oxygenation as measured by two portable, wireless near-infrared spectroscopy (NIRS) devices under resting and dynamic conditions. A recently developed low-cost NIRS device (MOXY) was compared against an established PortaMon system that makes use of the spatially resolved spectroscopy algorithm. The influence of increasing external pressure on tissue oxygen saturation index (TSI) indicated that both devices are stable between 2 and $20 \mathrm{mmHg}$. However, above this pressure, MOXY reports declining TSI values. Analysis of adipose tissue thickness (ATT) and TSI shows a significant, nonlinear difference between devices at rest. The devices report similar TSI (\%) values at a low ATT $(<7 \mathrm{~mm})$ (PortaMon minus MOXY difference is $+1.1 \pm 2.8 \%$ ) with the major subsequent change between the devices occurring between 7 and $10 \mathrm{~mm}$; at ATT values $>10 \mathrm{~mm}$ the difference remains constant $(-14.7 \pm 2.8 \%)$. The most likely explanation for this difference is the small source-detector separation $(2.5 \mathrm{~cm})$ in the MOXY resulting in lower tissue penetration into muscle in subjects with higher ATT. Interday test-retest reliability of resting TSI was evaluated on five separate occasions, with the PortaMon reporting a lower coefficient of variation ( $1.8 \%$ to $2.5 \%$ versus $5.7 \%$ to $6.2 \%$ ). In studies on male subjects with low ATT, decreases in the TSI were strongly correlated during isometric exercise, arterial occlusion, and incremental arm crank exercise. However, the MOXY reports a greater dynamic range, particularly during ischemia induced by isometric contraction or occlusion ( $\Delta 74.3 \%$ versus $\Delta 43.7 \%$; hyperemia MAX-occlusion MIN). This study shows that in this subject group both MOXY and PortaMon produce physiologically credible TSI measures during rest and exercise. However, the absolute values obtained during exercise are generally not comparable between devices unless corrected by physiological calibration following an arterial occlusion. ๑ The Authors. Published by SPIE under a Creative Commons Attribution 3.0 Unported License. Distribution or reproduction of this work in whole or in part requires full attribution of the original publication, including its DOI. [DOI: 10.1117/1.JBO.23.1.015007]
\end{abstract}

Keywords: near-infrared spectroscopy; reliability; muscle oxygen saturation; isometric exercise.

Paper 170339RR received May 26, 2017; accepted for publication Jan. 4, 2018; published online Jan. 24, 2018.

\section{Introduction}

The use of wearable devices or wearable biosensors, which allow the continuous monitoring of physiological signals, is important for better monitoring of active lifestyles and the diagnosis and treatment of diseases. ${ }^{1}$ Near-infrared spectroscopy (NIRS) is a noninvasive technique capable of providing valuable functional insights into skeletal muscle oxidative metabolism in vivo during exercise, in healthy and clinical populations. ${ }^{1}$ NIRS directly measures the oxygen-dependant absorption of hemoglobin $(\mathrm{Hb})$ in the microcirculation blood vessels (i.e., arterioles, capillaries, and venules) and myoglobin $(\mathrm{Mb})$ in the muscle cytoplasm. ${ }^{2}$

Dependent on the NIRS device and illumination type, it is possible to retrieve absolute and/or relative concentration values of oxyhemoglobin $\left(\mathrm{O}_{2} \mathrm{Hb}\right)$ and deoxyhemoglobin $(\mathrm{HHb})$, and a derived parameter, total-hemoglobin $\left(\mathrm{tHb}=\mathrm{O}_{2} \mathrm{Hb}+\mathrm{HHb}\right){ }^{3}$ These values are in a large part representative of tissue capillaries, arterioles, and venules because, in larger blood vessels, NIR light is fully absorbed by the high hemoglobin concentration. ${ }^{4}$ A further measurement, tissue saturation index (TSI) represents

*Address all correspondence to: Chris J. McManus, E-mail: cmcman @essex. ac.uk the ratio of oxygenated $\left(\mathrm{O}_{2} \mathrm{Hb}+\mathrm{O}_{2} \mathrm{Mb}\right)$ concentration to total $(\mathrm{tHb}+\mathrm{tMb})$ concentration, reporting on the dynamic balance between $\mathrm{O}_{2}$ supply and $\mathrm{O}_{2}$ consumption. Taken as a whole, the range of muscle NIRS parameters can provide information regarding changes in key dynamic physiological indicators, such as blood flow, oxygen extraction, and oxygen consumption; muscle NIRS studies are therefore relevant in the evaluation of exercise performance, exercise tolerance, ${ }^{1}$ and exercise training adaptation that can influence the balance between muscle $\mathrm{O}_{2}$ delivery and muscle utilization. ${ }^{5}$

The use of NIRS as a research tool is well established for studying exercise physiology changes in groups of subjects. ${ }^{1,6}$ Sports science applications could include monitoring changes in muscle blood flow and oxidative capacity as a function of training. ${ }^{4}$ In support of this, positive peripheral muscle oxygenation adaptations have been reported as a consequence of cycling sprint interval training, demonstrating that NIRS has the potential to assess the effectiveness of training interventions at the level of the individual athlete. ${ }^{7}$ To be effective outside the research laboratory, in an applied exercise environment, there is a requirement for low-cost, portable NIR technology with telemetric capability with the ability to withstand the rigors of sporting environments such as in swimming, ${ }^{8}$ short track speed skating, ${ }^{9}$ and trail running. ${ }^{10}$ 
Past studies comparing NIRS monitors have highlighted discrepancies in the values obtained at rest and during exercise in human ${ }^{11-14}$ and animal ${ }^{15}$ systems. The differences between monitors are likely to be in part associated with the measurement site and spacing of the probes. ${ }^{16,17}$ The specific optical properties of the system, whether laser, white light or light-emitting diode (LED)-based, as well as the specific NIR wavelengths monitored, could also contribute to differences between systems. These physical properties are most likely reflected in time resolution, signal:noise, and reproducibility. However, the absolute numbers of the derived parameters are highly dependent on the algorithms used in the calculations. In terms of measurements of TSI, these methods attempt to remove or correct for the contributions of light scattering and then fit the remaining optical density changes to the known NIR spectra of the hemo(myo)globin chromophores. Assumptions are also made about light transport generally assuming the diffusion model.

Time of flight or frequency domain measurements can be used to measure absolute chromophore concentrations, but such devices are currently unlikely to be cheap enough, or portable enough, to be of use for routine physical activity measurements. Manufacturers have therefore focussed on developing methods that only require continuous wave light detection, relying on the use of multiwavelength or multidistance analysis to correct for the scattering. Multiwavelength measures, e.g., using second derivative spectroscopy, ${ }^{18,19}$ are now rarely used. However, the use of multidistance methods has significantly expanded in recent years. In a recent review, only 1 of 13 current devices measured optical density at a single source:detector distance. ${ }^{1}$

In general, multidistance NIR systems correct for light scattering by one of two methods. The most common method used in historical terms is spatially resolved spectroscopy (SRS) This measures the slope of the optical density change as a function of multiple distances. Theoretically, the difference in these distances should be small compared to the average source:detector separation. This enables the calculation of scaled absorption coefficient $\left(\mu_{\mathrm{a}}\right)$ measures, which can allow absolute values of chromophore ratios, such as tissue oxygen saturation. ${ }^{20}$ The major commercial incarnations of the SRS system historically are the Hamamatsu NIRO and the Artinis OxyMon systems. Alternative spatial resolution (SR) methods use a larger separation among measurement sites; light transport models assuming the shorter distance predominantly measures surface tissues and the longer distance deeper tissues are used to calculate a tissue oxygen saturation for the deeper (muscle/brain) region of interest. $^{21}$ The predominant commercial incarnation of this SR method historically is the Medtronic INVOSTM and more recently the FORE-SIGHT ELITE ${ }^{\circledR}$ and the Nonin EQUANOX ${ }^{\mathrm{TM}}$ systems.

Although one can assume that two different devices both using the SRS measurements will generally report similar muscle oxygen saturations, the same cannot be assumed between devices using SR methods, given the variety of algorithms in use, ${ }^{22}$ nor of course between an SRS device and an SR device. ${ }^{23}$ SRS systems have been widely used in exercise physiology and sports science. ${ }^{1,3}$ Recently, a new low-cost SR-based device, the MOXY monitor, has been marketed to athletes as a training tool to inform the user of exercise intensity. ${ }^{10,24}$ The PortaMon, a widely used, portable, SRS oximeter has previously demonstrated a high level of reliability at rest ${ }^{25}$ and during isotonic, multijoint resistance exercise. ${ }^{26}$ Conversely, no studies investigating the test-retest reliability of the MOXY device have been conducted.
It would be helpful if users of NIRS devices could be confident that the extensive historical SRS literature on exercise physiology and sports science can be used to help inform their use of this new SR device. The purpose of the study was therefore to compare these two portable, wireless NIRS devices under a variety of resting and dynamic conditions. These conditions include (1) the influence of varying external pressure at rest, (2) test-retest reliability and sensitivity at rest, (3) during isometric leg extension exercise, (4) during dynamic arm-crank exercise, and (5) during an arterial occlusion.

\section{Methods}

\section{$2.1 \quad$ NIRS Devices}

The two NIRS devices used in the assessment of muscle oxygen saturation include the PortaMon (PortaMon, Artinis, Medical System) and MOXY muscle monitor (Fortiori Design LLC).

The PortaMon is a compact $(83 \times 52 \times 20 \mathrm{~mm})$ and lightweight (84 g) NIRS system. It is a dual-wavelength (760 and $850 \mathrm{~nm}$ ), continuous wave system, containing three pairs of LEDs in an SRS configuration with a source-detector spacing of 30,35 , and $40 \mathrm{~mm}$. The device simultaneously uses the modified Beer-Lambert law and SRS methods to calculate the absolute concentration of tissue oxy $\left(+\right.$ myo hemoglobin, $\left(\mathrm{O}_{2} \mathrm{Hb}\right)$, deoxyhemo(+myo)globin $(\mathrm{HHb})$, and total hemo(+myo)globin $(\mathrm{tHb})$. As such, tissue oxygen saturation (TSI) is expressed in \% and calculated as $\left[\mathrm{O}_{2} \mathrm{Hb}\right] /\left(\left[\mathrm{O}_{2} \mathrm{Hb}\right]+[\mathrm{HHb}]\right) \times 100$, which is the ratio of $\mathrm{HbO}_{2}$ to $\mathrm{tHb}$. TSI reflects the dynamic balance between $\mathrm{O}_{2}$ supply and consumption. During all testing, the system was connected to a personal computer via Bluetooth ${ }^{\mathrm{TM}}$ technology for data acquisition $(10 \mathrm{~Hz})$, analog-to-digital conversion and subsequent analysis.

The MOXY is a self-contained, compact $(61 \times 44 \times 21 \mathrm{~mm})$, and lightweight (48 g) NIRS system. It employs four wavelengths of NIR light at 680, 720, 760, and $800 \mathrm{~nm}$ (Schmitz, 2015) and the sensor contains a single LED and two detectors in placed 12.5 and $25.0 \mathrm{~mm}$ from the source. The MOXY device reports a change in total tissue hemo(+myo)globin (tHb) and tissue oxygenation (TSI). During all testing, the system was connected to a personal computer via a commercially available software program (Peripedal (C) to provide a graphic display of the data. Data acquisition $(2 \mathrm{~Hz})$ was obtained from the sensors internal memory.

\subsection{Testing the Influence of External Pressure}

In order to minimize movement artifacts when fixing NIRS devices to the tissue, a variety of supportive bandages or neoprene sleeves are generally used. These sleeves apply external pressure to the device and the surrounding limb area under compression. The influence of external pressure on NIRS derived values is largely undiscussed in the literature, yet if found that TSI data differ due to varying low external pressure this is of importance for both the research and applied user community.

A repeated-measures experimental design was used to determine the influence of external pressure on the reliability of two portable NIRS devices. Four recreationally active male participants ([mean $\pm \mathrm{SD}]$ age $22.75 \pm 5.56$ years, body mass $78.03 \pm 4.38 \mathrm{~kg}$, height $1.86 \pm 0.1 \mathrm{~m}$, vastus lateralis (VL) skinfold thickness $8.30 \pm 2.51 \mathrm{~mm}$ ) volunteered to participate in this study. None of the participants had known health problems or any lower extremity muscle or joint injury. Participants 
were asked not to perform any strenuous exercise or consume caffeine for at least $24 \mathrm{~h}$ prior to visiting the laboratory.

Participants were required to visit on one occasion. All testing was performed in a controlled laboratory environment $\left(\sim 20^{\circ} \mathrm{C}\right)$ at the University of Essex Clinical Physiology Laboratory. Upon arrival, following the collection of descriptive measures, participants performed $10 \mathrm{~min}$ of supine rest on a medical examination bench. The first portable NIRS device was then positioned on the belly of the vastus lateralis, midway between the greater trochanter and the lateral epicondyle of the femur. NIRS devices were placed precisely on the VL, whereby the middistance between the closest emitting diode and detector for each device was aligned with the VL location. To ensure the optodes and detector did not move relative to the participants' skin, the device was fixed into position using a waterproof adhesive tape. In addition, bodily hair at or around the sensor placement area was removed from the skin and participants were asked not to moisturize the area on the day of testing. A portable pressure monitor (PicoPress, MircoLabs, Italy) was used to assess the external pressure applied to the leg. An air-filled bladder was placed on the medial aspect of the thigh, at the same vertical alignment to the NIRS device location. A surgical marker pen was used to mark the position of NIRS device and bladder placement in order to identify any movement during testing and ensure the second NIRS device was placed at the same location. ${ }^{7}$ A black neoprene thigh sleeve (Mueller, Wisconsin) was wrapped around the leg to secure the NIRS device in place firmly against the skin and also ensure that no external light would be received by the device detectors.

Incremental external pressure $(\mathrm{mmHg})$ was applied to the surrounding area, encompassing the NIRS device and pressure monitor bladder, using an aneroid sphygmomanometer (Welch Allyn Inc., New York, New York) connected to a thigh blood pressure cuff (Accoson Works, Essex, United Kingdom). All measurements were recorded in a supine position; with the order of NIRS device application counterbalanced. Initial external pressure applied was $2 \mathrm{mmHg}$ as a result of the neoprene strapping, prior to inflating a blood pressure cuff in a stepwise manner.

Initially, the investigator visually observed the live NIRS data on a personal computer until a stable value was achieved and collected $60 \mathrm{~s}$ of data. Stability was defined as a TSI variation $<2 \%$ over $30 \mathrm{~s} .{ }^{27}$ This procedure was repeated for all NIRS devices. After 4 min of baseline data collection with the neoprene strap applying $2 \mathrm{mmHg}$, the sphygmomanometer was inflated to elicit a pressure of $5 \mathrm{mmHg}$, with $5 \mathrm{mmHg}$ increments every $4 \mathrm{~min}$ thereafter, up to and including $30 \mathrm{mmHg}$. The data acquired from the PortaMon system were downsampled from 10 to $2 \mathrm{~Hz}$ to allow for comparison with the MOXY system. Values are reported as average absolute TSI, calculated from the final $60 \mathrm{~s}$ of each $4 \mathrm{~min}$ period. Descriptive statistics are presented as mean (TSI) unless otherwise stated. A simple linear regression was then used to calculate slopes of the NIRS device versus external pressure. Subsequently, the TSI of PortaMon minus MOXY at incremental external pressures could be fitted by a sigmoid curve. All analyses were performed using Graphpad Prism 7 (Graphpad Software, San Diego, California).

\subsection{Comparing Devices at Rest}

Muscle oxygenation of the dominant vastus lateralis muscle of each participant was measured with two MOXY (MOXY ${ }^{1}$ and $\mathrm{MOXY}^{2}$ ) and two PortaMon (PortaMon ${ }^{1}$ and PortaMon ${ }^{2}$ ) devices to assess between and within-device variation. Twentyone male ([mean $\pm \mathrm{SD}]$ age $22.33 \pm 4.84$ years, body mass $78.24 \pm 10.62 \mathrm{~kg}$, height $1.79 \pm 0.1 \mathrm{~m}$, VL skinfold thickness $11.59 \pm 5.09 \mathrm{~mm}$ ) and nine female participants (age $21.67 \pm$ 2.40 years, body mass $65.14 \pm 7.44 \mathrm{~kg}$, height $1.69 \pm 0.04 \mathrm{~m}$, VL skinfold thickness $26.36 \pm 4.77 \mathrm{~mm}$ ) volunteered to participate in this study. The experimental protocol involved a single visit to the laboratory. Upon arrival, participants performed 10 min of supine rest on a medical examination bench. The first of four NIRS devices was positioned on the vastus lateralis muscle (as described in Sec 2.2), and all measurements were recorded in a supine position. Using a portable pressure monitor, care was taken to ensure the same external pressure $(\sim 8 \mathrm{mmHg})$ was exerted by the neoprene strapping surrounding the NIRS device, during each condition. Device order was counterbalanced using a Latin square design. The investigator visually observed the live NIRS data on a personal computer until a stable value was achieved (minimum $2 \mathrm{~min}$ ) and collected $60 \mathrm{~s}$ of data. This procedure was repeated for all four NIRS devices.

Descriptive statistics are presented as mean \pm SD unless otherwise stated. A one-way analysis of variance (ANOVA) was used to compare mean values of resting TSI between the four NIRS devices. A Tukey's range test was used for post hoc multiple comparisons. Subsequently, an unpaired $t$-test was performed to compare the combined average of two similar devices at rest (Mean TSI (PortaMon + PortaMon) versus mean TSI (MOXY + MOXY). Accuracy was calculated as the mean absolute difference, using Bland-Altman agreement plots $( \pm 2 \mathrm{SD})$ between resting TSI. A 95\% confidence interval of the mean (95\%CL) is also presented for each case. The difference between devices was calculated as the mean TSI of PortaMon minus MOXY. A Pearson's product-moment correlation analysis was used to compare the association between resting values of TSI and quadriceps skinfold thickness [adipose tissue thickness $(\mathrm{ATT})=0.5 \times$ mean skinfold thickness]. Significance was set at $P<0.05$. All statistical analyses were performed using Graphpad Prism 7 (Graphpad Software, San Diego, California).

\subsection{Test-Retest Reliability and Sensitivity of Devices at Rest}

Five male participants ([mean \pm SD] age $21.2 \pm 2.7$ years, body mass $71.5 \pm 7.7 \mathrm{~kg}$, height $1.78 \pm 0.1 \mathrm{~m}$, and VL skinfold thickness $11.3 \pm 5.3 \mathrm{~mm}$ ) undertook repeated resting measures assessing vastus lateralis TSI (\%) for a total of five occasions. Each visit to the laboratory was separated by at least $24 \mathrm{~h}$, thereby providing a method of assessing interday test-retest reliability of four NIRS devices (MOXY ${ }^{1}, \mathrm{MOXY}^{2}$, PortaMon $^{1}$, and PortaMon ${ }^{2}$ ). Upon arrival on each occasion, participant's vastus lateralis was identified as described in Sec 2.2. Neoprene strapping was carefully tightened around the NIRS device to ensure a pressure of $8 \mathrm{mmHg}$ was exerted. The same researcher attached each device on every occasion to minimize intertester variability. After $10 \mathrm{~min}$ supine rest, the investigator visually observed the live NIRS data on a personal computer until a stable value was achieved (minimum $2 \mathrm{~min}$ ) and collected $60 \mathrm{~s}$ of data. This procedure was repeated for all four NIRS devices using a Latin square design.

Descriptive statistics are presented as mean \pm SD unless otherwise stated. The mean participant TSI for each NIRS device was used as an indicator of test-retest reliability. ${ }^{28}$ The coefficient of variation $(\mathrm{CV})$ was calculated as a characteristic of the 
performance of within-subject evaluation, ${ }^{29}$ with a $\mathrm{CV}$ below $5 \%$ to $10 \%$ considered as acceptable absolute reliability. ${ }^{26}$

All statistical analyses were performed using Microsoft Excel and GraphPad Prism 7.

\subsection{Comparing Devices During Isometric Exercise}

A repeated isometric quadriceps contraction exercise protocol was implemented to compare the delta $(\Delta)$ TSI between four NIRS devices (MOXY ${ }^{1}$, MOXY $^{2}$, PortaMon $^{1}$, and PortaMon ${ }^{2}$ ). The same five male participants ([mean \pm SD] age $21.2 \pm 2.7$ years, body mass $71.5 \pm 7.7 \mathrm{~kg}$, height $1.78 \pm 0.1 \mathrm{~m}$, and VL skinfold thickness $11.3 \pm 5.3 \mathrm{~mm}$ ) who underwent the testretest reliability assessment at rest (see Sec. 2.4), participated in a quadriceps isometric contraction task. Participants were asked not to perform any strenuous exercise or consume caffeine for at least $24 \mathrm{~h}$ prior to visiting the laboratory.

During the initial visit, the participant's maximal voluntary isometric contraction (MVC) was determined. Prior to the isometric contraction task, a nonspecific warm-up on a cycle ergometer (Monark, 824E) was completed, before performing an MVC of the knee extensors using an isokinetic dynamometer (KinCom, Chattanooga Inc., Tennessee). Participants were positioned upright on the dynamometer ensuring the knee joint (lateral femoral epicondyle) and dynamometer axes were accurately aligned and stabilized using leg, waist, and chest straps to minimize trunk movement during testing. The seat height and distance from the axis were recorded to ensure accurate repeat positioning. The dominant leg was then fixed at a 60 deg angle and three submaximal repetitions of a 5-s continuous quadriceps isometric contraction were performed. Participants then performed three maximal contractions interspersed with a 60-s rest period. The best of the three contractions was considered the maximal isometric force and values for $30 \%$ and $50 \%$ MVC were then calculated.

During the second visit, after resting data were collected from all four NIRS devices (see Sec. 2.3), the final NIRS device was left attached to the participant's leg for the duration of the quadriceps isometric contraction task. Participants were positioned on the dynamometer (as described above) with no warm-up. After a stable pretest TSI value was attained, the participants were instructed to perform two 30-s sustained isometric quadriceps contractions to the previously defined values of their MVC (30\% and 50\%) interspersed by a 3-min rest period. TSI was continuously monitored throughout the procedure. This procedure was repeated for visits 3, 4, and 5 using a different NIRS device during the isometric contraction task.

Due to potential differences in resting values for the two devices $(2.3 ; 2.4)$, TSI was reported as a change from pretest (60 $\mathrm{s}$ averaging before each test). Maximum and minimum TSI values were calculated as a three-second average surrounding the highest and lowest values during each of the two isometric contractions. The $\Delta$ in TSI was therefore calculated as the maximum value minus the minimum value during each $30 \mathrm{~s}$ contraction, e.g., TSI Max - TSI Min $=\Delta$ TSI $^{8}$

Descriptive statistics are presented as mean \pm SD unless otherwise stated. A one-way analysis of variance (ANOVA) was used to compare mean values of $\Delta$ TSI between the four NIRS devices. A Tukey's range test was used for post hoc multiple comparisons. ICC was calculated to compare between-device reliability at $30 \%$ and $50 \% \mathrm{MVC}(\Delta \mathrm{TSI})$. Accuracy was calculated as the mean absolute difference, using Bland-Altman agreement plots $( \pm 2 \mathrm{SD})$ between resting TSI. A $95 \%$ confidence interval of the mean $(95 \% \mathrm{CI})$ is also presented for each case. The difference between devices was calculated as the mean TSI of MOXY minus PortaMon. All statistical analyses were performed using Graphpad Prism 7 (Graphpad Software, San Diego, California). The studies undertaken all received ethical approval from the University of Essex ethical committee and participants provided written informed consent before inclusion.

\subsection{Comparing Devices During Dynamic Exercise and Occlusion}

An incremental asynchronous arm-crank exercise protocol, followed by a 5-min arterial occlusion, was undertaken to compare TSI values between two NIRS devices $\left(\mathrm{MOXY}^{1}\right.$ versus PortaMon $\left.{ }^{1}\right)$. Based on the effect size observed between NIRS devices during isometric exercise at 50\% MVC, a power analysis for a dependant sample $t$-test was conducted using $\mathrm{G}^{*}$ Power to determine a sufficient sample size using an alpha of 0.05 , a power of 0.80 , a large effect size $(d z=2.9)$, and two tails. ${ }^{30}$ Based on the aforementioned assumptions, the desired sample size is 4 . We elected to recruit an additional two participants to allow for participants terminating the arterial occlusion early due to the sensation of pain. Six male participants ([mean \pm SD] age $23.7 \pm 4.1$ years, body mass $73.3 \pm 5.2 \mathrm{~kg}$, ATT $2.7 \pm 1.0 \mathrm{~mm}$ ) undertook the exercise and occlusion task, requiring two separate visits to the laboratory, separated by 3 to 7 days. On arrival, following basic anthropometrical measures, the participant confirmed their dominant arm (all participants' self-reported right hand dominance) and ATT was assessed at the muscle belly of the biceps brachii, $8 \mathrm{~cm}$ above the midarm fold. The first NIRS device was positioned on the biceps brachii, where the $8 \mathrm{~cm}$ marker was used to determine the midpoint between the device emitter and detectors. The biceps brachii were chosen as previous literature has reported this muscle eliciting the greatest decrease in tissue oxygenation index during arm cranking compared with the triceps brachii, brachioradialis, and anterior deltoid. ${ }^{5}$ A portable pressure monitor was used to ensure a similar external pressure was exerted by the black crepe bandage used to hold the device in place. Subsequently, the participant's sat upright, with hands in a rested position until a stable TSI value was achieved (minimum $2 \mathrm{~min}$ ) and a resting baseline was obtained over a 5 -min period.

Prior to arm cranking, participants were positioned with the crank axis aligned to shoulder height, at a distance that allowed a slight bend in the elbow when arms were extended. A chest strap was applied to minimize trunk movement during testing and seat/crank height were recorded to ensure accurate repeat testing. Participants were required to arm-crank at $60 \mathrm{rpm}$ beginning at 0 Watts $(\mathrm{W})$ and increasing by $25 \mathrm{~W}$ every $2 \mathrm{~min}$. The exercise end-point was determined when cadence fell below $60 \mathrm{rpm}$ for 3 consecutive seconds.

Following completion of the incremental arm-crank exercise task, participants remained seated with hands in a rested position for 5 min prior to an arterial occlusion. To enable a physiological calibration of raw TSI values by identifying the participant's notional minimum and maximum TSI, an arterial occlusion was applied for 5 min using an automated pneumatic cuff inflator (Hokanson, Bellevue) positioned at the proximal aspect of the humerus, applying 295 to $305 \mathrm{mmHg}$ of pressure. After a 5-min occlusion, the cuff was released and participant's continued to rest for a further $5 \mathrm{~min}$ period. This procedure was repeated for the second visit using the alternative NIRS device. Although we cannot be sure that this value exactly corresponds 
to complete deoxygenation of hemoglobin and myoglobin, in all subjects this time was sufficient to result in a stable minimum in saturation value significantly lower than any observed during exercise.

Initially, the data acquired from the PortaMon system were downsampled from 10 to $2 \mathrm{~Hz}$ to match that of the MOXY system. Following this, both device data-sets were smoothed by calculating the $3 \mathrm{~s}$ moving average at $2 \mathrm{~Hz}$. To normalize the NIRS signal and achieve a physiological calibration, the average value of the final $30 \mathrm{~s}$ was used to determine notional $0 \%$ oxygenation (calibration) and the $30 \mathrm{~s}$ peak hyperemic response upon release of the cuff was used to indicate notional $100 \%$ oxygenation (we use the term "notional" as we cannot be absolutely sure that the lower value could not be decreased further by adding an ischemic exercise component, not that the upper value could not be increased by hyperoxia and/or methods to increase blood flow or decrease oxygen consumption; however, the current physiological calibration has the advantage of being relatively easy to implement and in common use in the field). The $\Delta$ in TSI during the occlusion task was calculated as the maximum value minus the minimum value, identified from the onset of the cuff inflating to the period following the hyperemic response upon cuff release.

During the incremental test, TSI was established for each power output as the final $30 \mathrm{~s}$ average for each stage. These values were subtracted from the resting baseline TSI to provide a delta $(\Delta)$ raw TSI and subsequently converted to a calibrated $(\%$ calibration) value.

Descriptive statistics are presented as mean [95\% CI] unless otherwise stated. Paired $t$-tests were used to compare performance outcomes of the arm-crank test and compare device minimum, maximum, and delta values for raw (TSI) and calibrated (\% calibration) data during the occlusion. Furthermore, a repeated measure ANOVA was conducted to compare values obtained during the incremental arm-crank test for each power output. Significant $F$ ratios were examined on a post hoc basis using an uncorrected Fisher's LSD test. Where data were not obtained for all participants at peak power output (i.e., only three participants completed the $100-\mathrm{W}$ stage), a paired $t$-test was conducted on the smaller sample size to compare differences between devices. The incremental data are reported as the change from resting baseline in both raw ( $\Delta$ TSI) and calibrated units ( $\Delta$ calibration $\%)$. A simple linear regression was then used to calculate slopes of the NIRS device versus power output.

\section{Results}

\subsection{Testing the Influence of External Pressure}

There was a significant correlation between TSI and external pressure for both MOXY and PortaMon devices [Fig. 1(a)], in both cases showing a drop in TSI with increasing pressure. The slope was significantly steeper for the MOXY than the PortaMon (a slope of $-0.38 \pm 0.09 \%$ TSI per $\mathrm{mmHg}$ for the MOXY compared to $-0.08 \pm 0.03$ for the PortaMon). The PortaMon reports a lower resting TSI (\%) at low pressure $(<20 \mathrm{mmHg}$ ); with the major subsequent change between the devices occurring between 20 and $30 \mathrm{mmHg}$ [Fig. 1(b)]. Therefore, to decrease variability, the subsequent studies were all undertaken at lower than $<20 \mathrm{mmHg}$, with a target external pressure of $8 \mathrm{mmHg}$ when participants were supine.

\subsection{Comparing Devices at Rest}

Resting TSI of male and female participants for four NIRS devices is provided in Table 1. A one-way ANOVA of mean resting TSI among the four NIRS devices for female participants demonstrated a significant difference $(P<0.05)$, with post hoc multiple comparisons showing a significant difference between MOXY and PortaMon devices (MOXY ${ }^{1}$ versus PortaMon ${ }^{1}$; MOXY $^{1}$ versus PortaMon ${ }^{2}$; MOXY ${ }^{2}$ versus PortaMon ${ }^{1}$; MOXY ${ }^{2}$ versus PortaMon ${ }^{2}=P<0.05$ ). Conversely, no difference was found between devices for male participants.

As no significant difference was found at rest between either MOXY $^{1}$ versus MOXY ${ }^{2}$ or PortaMon ${ }^{1}$ versus PortaMon ${ }^{2}$, the combined mean TSI for MOXY (MOXY ${ }^{1}$ and MOXY ${ }^{2}$ ) and PortaMon (PortaMon ${ }^{1}$ and PortaMon ${ }^{2}$ ) at rest for male and female participants (Table 1) was compared using an unpaired $t$-test. Mean female MOXY TSI $(90.9 \pm 5.1 \%)$ was significantly higher than the PortaMon $(76.7 \pm 2.6 \%)[t(16)=7.4, P<$ $0.05]$; however, no significant difference was observed in male participants between the MOXY $(74.3 \pm 12.8 \%)$ and PortaMon $(72.2 \pm 3.5 \%)$ devices.

At rest, Bland-Altman plots of male participants [Fig. 1(c)] suggest the MOXY device reports a higher saturation value at higher resting TSI and a lower value at lower resting TSI. So, the lack of a significant difference of the mean value masks a real measurement difference between the devices even in male subjects. Bland-Altman plots of resting female participants [Fig. 1(d)] illustrate a consistently higher TSI with the MOXY device versus PortaMon, regardless of average TSI, consistent with the difference in the mean values.

Vastus lateralis skinfold thickness (mm) and resting TSI (\%) are highly correlated using the combined mean TSI MOXY $(r=0.70, P<0.01)$ and PortaMon device $(r=0.73, P<0.01)$. Linear regression of VL skinfold thickness and TSI [Fig. 2(a)] shows the difference between the slopes (MOXY versus PortaMon) are significantly different $[F(1,54)=11.97, P=$ $0.001]$. The MOXY device shows a regression slope $(y=$ $1.12 * x+61.22)$, steeper than that of the PortaMon $(y=$ $0.34 * x+68.16)$. This indicates that as skinfold thickness increases, the rise in TSI is greater with the MOXY. The difference between the two values is nonlinear with respect to ATT [Fig. 2(b)]. The PortaMon and MOXY devices report similar TSI $(\%)$ values at a low ATT $(<7 \mathrm{~mm})$; with the major subsequent change between the devices occurring between 7 and $10 \mathrm{~mm}$; at ATT values $>10 \mathrm{~mm}$ the difference remains constant. The data fit well to a sigmoidal dose response relationship. At the lowest ATT, the (PortaMon minus MOXY) difference is $+1.1 \pm 2.8 \%$; (mean + SEM), at the highest ATT the difference is $-14.7 \pm 2.8 \%$; the $50 \%$ change occurs at $8.5 \pm 0.6 \mathrm{~mm}$ ATT.

\subsection{Test-Retest Reliability of Devices at Rest}

The CV for all NIRS devices are listed in Table 2. The mean CV values were lower for the PortaMon $(<2.5 \%)$ when compared with the MOXY devices (5.7\% to 6.2\%); however, both devices were below the acceptable threshold of $10 \%$. The SD of the individual devices highlights a smaller measurement variation when using the PortaMon, suggesting that the intersubject variation across repeated measures is less than the MOXY device.

\subsection{Comparing Devices During Isometric Exercise}

Mean torque of $30 \%$ and $50 \%$ MVC was $256.9 \pm 43.5$ and $428.2 \pm 72.5 \mathrm{~N}$, respectively. A one-way ANOVA of $\Delta \mathrm{TSI}$ 
(a)

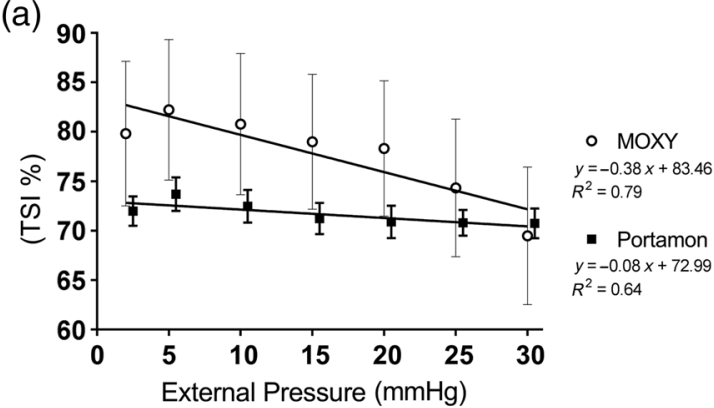

(c)

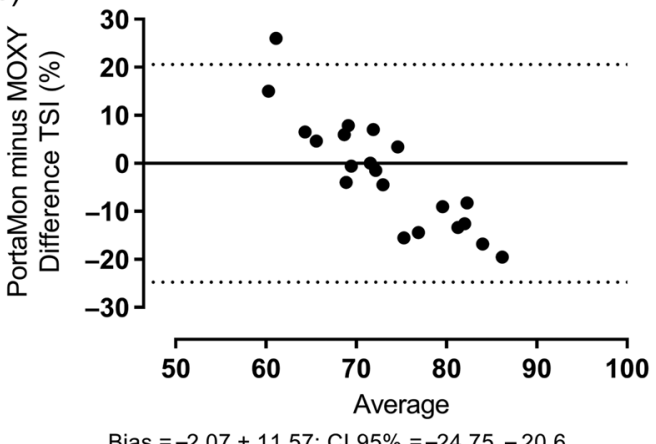

(b)

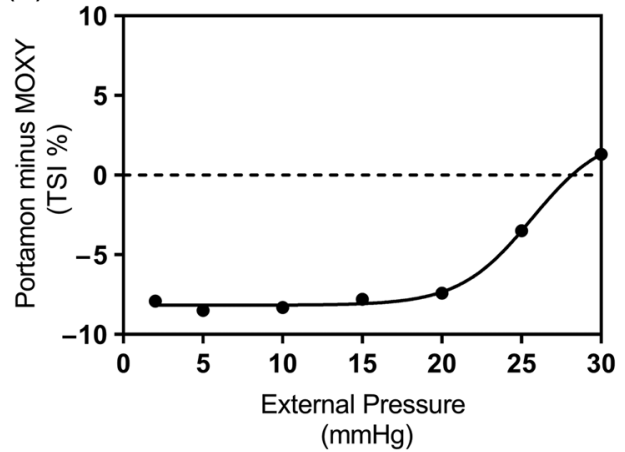

(d)

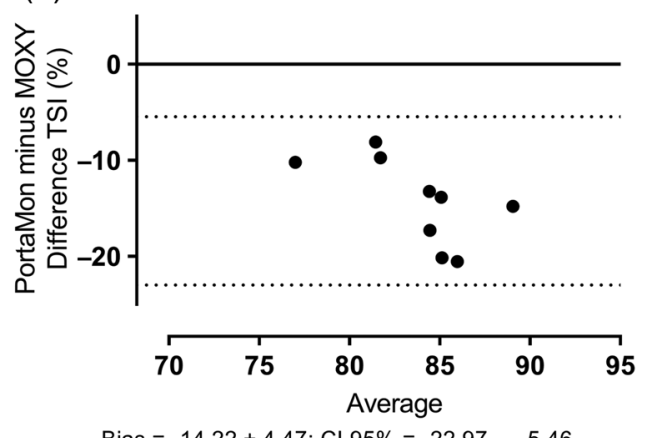

Fig. 1 (a) Linear regression showing TSI as a function of external pressure $(\mathrm{mmHg})(\mathrm{mean} \pm \mathrm{SEM})$, (b) PortaMon minus MOXY (TSI) as a function of external pressure ( $\mathrm{mmHg}$ ). Data fit using nonlinear regression to a sigmoidal curve for illustrative purposes and (c) Bland-Altman plots of resting TSI of males $(n=21)$ and (d) females $(n=9)$ using the combined average of MOXY and PortaMon devices. Each plot represents the difference versus the average of the two devices. Dotted lines represent the $\pm 2 S D$ limits of agreement.

Table 1 Male and female mean TSI of four NIRS devices at rest.

\begin{tabular}{|c|c|c|}
\hline & Male TSI (\%) & Female TSI (\%) \\
\hline & Mean \pm SD $[95 \% \mathrm{Cl}]$ & Mean \pm SD $[95 \% \mathrm{Cl}]$ \\
\hline MOXY1 & $75.2 \pm 13.1[69.3,81.2]$ & $92.7 \pm 5.0^{*}[88.8,96.6]$ \\
\hline MOXY² & $73.3 \pm 12.8[67.5,79.2]$ & $89.1 \pm 6.3^{*}[84.3,94.0]$ \\
\hline PortaMon ${ }^{1}$ & $72.8 \pm 3.5[71.2,74.4]$ & $77.3 \pm 2.7[75.2,79.4]$ \\
\hline PortaMon $^{2}$ & $71.6 \pm 3.7[70.0,73.3]$ & $76.1 \pm 2.8[74.0,78.3]$ \\
\hline $\begin{array}{l}\text { Combined } \\
\text { MOXY }\end{array}$ & $74.3 \pm 12.8[68.5,80.1]$ & $90.9 \pm 5.1^{* \star}[87.0,94.9]$ \\
\hline $\begin{array}{l}\text { Combined } \\
\text { PortaMon } \\
\end{array}$ & $72.2 \pm 3.5[70.6,73.8]$ & $76.7 \pm 2.6[74.7,78.7]$ \\
\hline
\end{tabular}

*Significantly different from PortaMon ${ }^{1}$ and PortaMon ${ }^{2}(P>0.05)$. ** Significantly different to Combined PortaMon $(P>0.001)$.

during $30 \%$ and $50 \%$ MVC between the four NIRS devices demonstrated a significant difference $(P<0.05)$, with post hoc multiple comparisons showing a significant difference between MOXY and PortaMon devices (MOXY ${ }^{1}$ versus PortaMon ${ }^{1}$; MOXY $^{1}$ versus PortaMon ${ }^{2}$; MOXY ${ }^{2}$ versus PortaMon ${ }^{1}$; MOXY $^{2}$ versus PortaMon ${ }^{2}=P<0.05$ ). Table 3 displays the $\Delta$ TSI values.
No significant difference was found during the isometric quadriceps exercise between either $\mathrm{MOXY}^{1}$ and $\mathrm{MOXY}^{2}$ or PortaMon $^{1}$ and PortaMon ${ }^{2}$. As such, the combined group averaged data $(n=5)$ for MOXY $\left(\mathrm{MOXY}^{1}\right.$ and MOXY $\left.\mathrm{MO}^{2}\right)$ and PortaMon (PortaMon ${ }^{1}$ and PortaMon ${ }^{2}$ ) during the quadriceps isometric contraction task is shown as a composite graph in Fig. 3(a). The general trend was similar for all five participants among devices. For both systems, an immediate decrease in TSI at the onset of isometric contraction (30\% MVC) was observed. This recovered rapidly following contraction cessation and, in some cases, overshooting (a hyperaemic response). A larger decrease in TSI was observed at 50\% MVC and returning to resting at task cessation. Bland-Altman plots indicate that the MOXY reports consistently higher $\Delta$ TSI at both $30 \%$ [Fig. 3(b)] and 50\% [Fig. 3(c)] MVC, with a trend showing the greater the average $\Delta$ TSI, the greater the difference.

\subsection{Comparing Devices During Dynamic Exercise and Occlusion}

Peak performance measures, ATT, external pressure applied by the NIRS devices, TSI data during the incremental arm-crank test, and subsequent arterial occlusion are presented in Table 4. No significant differences were observed for peak power output or time to exhaustion between trials and external pressure was applied between 9 and $13 \mathrm{mmHg}$. During the arterial occlusion and the following reactive hyperaemia, significant differences were observed between NIRS devices for minimum, maximum, 

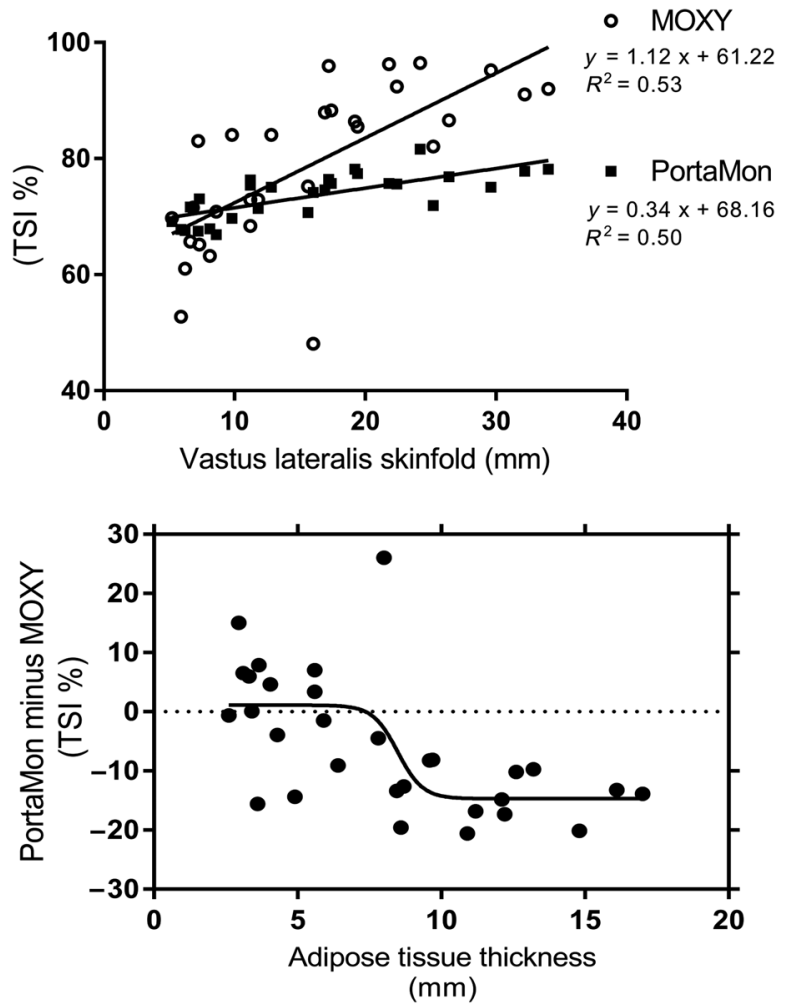

Fig. 2 (a) Relationship between mean TSI and vastus lateralis skinfold $(\mathrm{mm})$ using the combined average of MOXY and PortaMon devices and (b) PortaMon minus MOXY (TSI) as a function of ATT (mm). Data fit using nonlinear regression to a sigmoid dose response curve. and delta TSI. The MOXY device repeatedly reported a greater delta TSI when compared with the PortaMon $(p<0.01)$.

During the incremental arm-crank test, between-device TSI differences were observed at $25 \mathrm{~W}$ when comparing raw TSI values. In the postexercise hyperaemic period, the MOXY device also reported a significantly greater maximum value compared with the PortaMon $(p<0.01)$. However, these differences were not observed when the TSI range of the devices was calibrated to the min and max TSI associated with the arterial occlusion.

Despite the lack of significant differences seen at individual power values using the calibrated values, when the data are taken as a whole a subtle distinction can be observed. Figure 4 demonstrates the relationship between increasing power output and a progressive decrement in TSI. The regression slope was not significantly different $(P=0.18)$ between the MOXY and the PortaMon device for the raw TSI values [Fig. 4(a)] (a slope of $-0.16 \pm 0.02 \%$ TSI per $1 \mathrm{~W}$ for the MOXY compared to $-0.12 \pm 0.005$ for the PortaMon); however, the lines are not identical due to a significant difference in the intercepts $[F(1,7)=47.06, P=<0.01]$. A similar result is present when comparing the regression slopes of the calibrated values [Fig. 4(b)] between devices $(P=0.45)$, reporting similar decrements in TSI (\% calibrated) per $1 \mathrm{~W}$ increase (MOXY $=-0.23 \pm 0.03 \% ; \quad$ PortaMon $=-0.26 \pm 0.006 \%$ ), yet a statistically significant difference in the intercepts $[F(1,7)=12.95, P=<0.01]$. The difference in the intercepts appears to be due to a lower value of the MOXY after warmup compared to baseline (the $0-\mathrm{W}$ point is arm cranking with zero resistance compared to complete rest).

Table 2 Test-retest measures of device reliability (TSI) at rest.

\begin{tabular}{|c|c|c|c|c|c|c|c|c|}
\hline & \multicolumn{2}{|c|}{ MOXY' } & \multicolumn{2}{|c|}{ MOXY2 } & \multicolumn{2}{|c|}{ PortaMon ${ }^{1}$} & \multicolumn{2}{|c|}{ PortaMon ${ }^{2}$} \\
\hline & Mean \pm SD & CV (\%) & Mean \pm SD & CV (\%) & Mean \pm SD & CV (\%) & Mean \pm SD & CV (\%) \\
\hline Subject 1 & $52.9 \pm 4.4$ & 8.4 & $52.7 \pm 3.5$ & 6.7 & $67.9 \pm 1.0$ & 1.5 & $66.6 \pm 1.2$ & 1.7 \\
\hline Subject 2 & $87.8 \pm 4.8$ & 5.4 & $82.9 \pm 4.9$ & 5.9 & $74.7 \pm 1.9$ & 2.5 & $73.8 \pm 1.3$ & 1.8 \\
\hline Subject 3 & $56.9 \pm 5.5$ & 9.6 & $53.7 \pm 4.9$ & 9.2 & $66.0 \pm 1.1$ & 1.7 & $64.7 \pm 2.6$ & 4.1 \\
\hline Subject 4 & $87.2 \pm 3.1$ & 3.6 & $84.9 \pm 3.3$ & 3.9 & $71.3 \pm 0.8$ & 1.1 & $70.0 \pm 1.6$ & 2.3 \\
\hline Subject 5 & $71.8 \pm 2.7$ & 3.8 & $70.1 \pm 1.9$ & 2.7 & $72.7 \pm 1.6$ & 2.2 & $71.1 \pm 1.8$ & 2.6 \\
\hline \multicolumn{9}{|c|}{ Grouped data } \\
\hline CV (\%) & \multicolumn{2}{|c|}{$6.2[2.8,9.5]$} & \multicolumn{2}{|c|}{$5.7[2.5,8.8]$} & \multicolumn{2}{|c|}{$1.8[1.1,2.5]$} & \multicolumn{2}{|c|}{$2.5[1.3,3.7]$} \\
\hline
\end{tabular}

Note: CV, coefficient of variation [95\% confidence interval].

Table $3 \Delta$ change in TSI (\%) values during 30 s quadriceps isometric contraction task at $30 \%$ and $50 \%$ maximal voluntary contraction (MVC) of each NIRS device Mean \pm SD (range).

\begin{tabular}{ccccc} 
& MOXY1 & MOXY2 & PortaMon $^{1}$ & PortaMon $^{2}$ \\
\hline$\Delta$ TSI 30\% MVC (\%) & $36.5 \pm 7.3^{\mathrm{a}}(28.4-47.7)$ & $37.9 \pm 14.4^{\mathrm{a}}(15.2-55.2)$ & $9.4 \pm 4.4(5.0-16.3)$ & $8.3 \pm 4.8(2.9-15.7)$ \\
$\Delta$ TSI 50\% MVC (\%) & $57.3 \pm 14.9^{\mathrm{b}}(41.3-77.2)$ & $60.8 \pm 13.7^{\mathrm{b}}(42.3-75.9)$ & $14.2 \pm 2.3(11.7-16.7)$ & $14.8 \pm 4.3(9.9-19.7)$ \\
\hline
\end{tabular}

asignificant difference from PortaMon ${ }^{1}$ and PortaMon ${ }^{2}$ at $30 \%$ MVC $(P>0.05)$.

bSignificant difference from PortaMon ${ }^{1}$ and PortaMon 2 at $50 \%$ MVC $(P>0.05)$. 


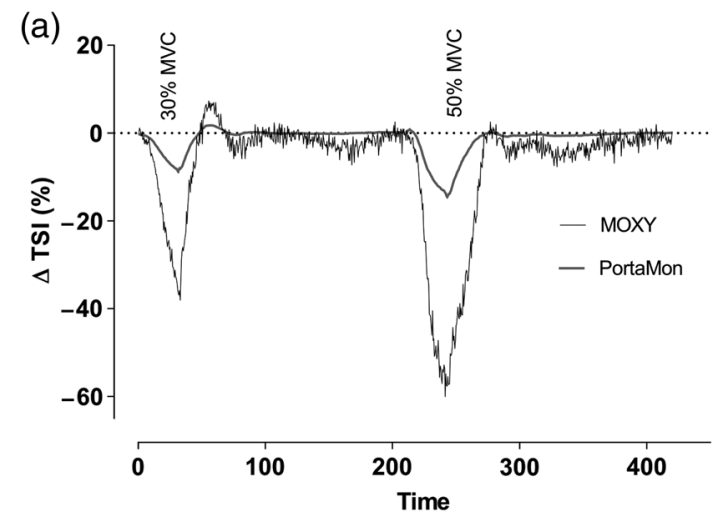

(b)

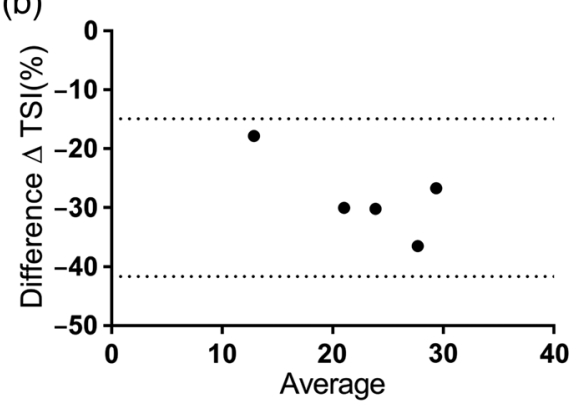

Bias $=-28.28 \pm 6.81 ; C L 95 \%=-41.63--14.93$

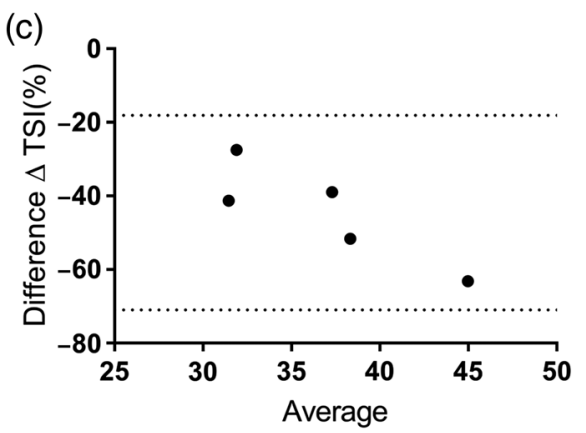

Bias $=-44.52 \pm 13.49 ;$ CL95\% $=-70.97--18.07$

Fig. 3 (a) Group changes in tissue saturation index ( $\Delta \mathrm{TSI})$ during $30 \%$ and $50 \%$ isometric MVC tasks and (b) Bland-Altman plots of $\Delta \mathrm{TSI}$ at $30 \% \mathrm{MVC}(n=5)$ and (c) $50 \% \mathrm{MVC}(n=5)$ using the combined average of MOXY and PortaMon devices. Each plot represents the difference versus mean $\Delta$ value measured as the average of the two MOXY devices compared to the two PortaMon devices. Dotted lines represent the $\pm 2 S D$ limits of agreement.

Figure 5 shows the raw and calibrated TSI traces during incremental arm cranking and the subsequent arterial occlusion for individual participants. It is evident from these figures that both devices follow a similar trend within individuals between both MOXY and PortaMon devices. Interestingly, following a rapid desaturation during the initial two stages of the armcrank test $(0$ and $25 \mathrm{~W})$, the TSI trace for participant 3 increased from $50 \mathrm{~W}$ to the end of the test. This physiological observation was reproduced by both devices for this individual on separate testing occasions.

\section{Discussion}

This current study compared two portable, wireless NIRS devices. While the measurement of muscle oxygen saturation correlated between the MOXY and PortaMon under a variety of resting and dynamic conditions, significant differences were noted, both with respect to device reliability and absolute values of saturation.

\subsection{Comparing Devices at Rest}

Light entering the surface above the vastus lateralis will pass through skin and adipose tissue layers before entering the muscle. It is generally assumed that NIR light reaching a detector has an effective penetration depth of $\sim 50 \%$ to $60 \%$ of the interoptode distance. ${ }^{31}$ Therefore, the PortaMon (maximum source:detector separation of $40 \mathrm{~mm}$ ) would be expected to detect more light from the deeper muscle tissue than the MOXY (maximum source:detector separation of $25 \mathrm{~mm}$ ). Even in the absence of differences in the measurement algorithm (discussed later), one would therefore not necessarily expect identical muscle oxygen saturation values for the two systems.

While the two devices did provide similar values for male subjects, in female subjects, the PortaMon reported a lower resting TSI when compared with the MOXY. We showed that this difference appeared to be consequent to the gender difference in adipose layer. Optical density is greater in subjects with a thin layer of adipose tissue. ${ }^{32}$ In SRS systems, the increased adipose layer results in a higher apparent \%TSI in female subjects. ${ }^{33}$ This effect is exaggerated in the MOXY. The smaller source: detector separation in the MOXY biases the light detected in favor of more surface tissues. It seems that the SR algorithm used is unable to completely correct for this effect. It is therefore even more important than usual in NIRS to measure and report on local body fat composition when using the MOXY, especially when comparing between subjects.

\subsection{Testing the Influence of External Pressure}

There were significant effects of the applied pressure on resting TSI, especially for the MOXY. At low external pressures (2 to $20 \mathrm{mmHg})$, the PortaMon reported a lower resting TSI $(\sim 8 \%)$ compared with the MOXY. As external pressure was increased above $20 \mathrm{mmHg}$, the difference between the two devices diminished. This difference was predominantly due to changes in the MOXY value, not the PortaMon. In particular, MOXY values showed a sharp decrease between 20 and $30 \mathrm{~mm} \mathrm{Hg}$. As external pressure is applied to the skins surface, arteriole, venule, and capillary intravascular hydrostatic pressure are equal in relation to the distance from the surface of the skin, ${ }^{34}$ occluding the flow of blood to the periphery. As a larger proportion of light detected in the MOXY comes from surface tissue it is likely that the MOXY is more sensitive to pressure-induced changes in the dermis and subcutaneous oxygenation and blood flow. Again, the SR algorithm used to measure muscle TSI is less effective than the SRS method in the PortaMon in correcting for the effects of differences in blood flow and oxygenation in surface tissues. This finding has an important implication for researchers when undertaking repeated measures using the MOXY device, particularly when the removal and reapplication of the NIRS device is required. Furthermore, athletes using similar devices to inform training intensity should standardize the external pressure applied to hold the instrument in place.

\subsection{Test-Retest Reliability of Devices at Rest}

This is the first study to report reliability data of the MOXY device. In the current study, we report absolute [the similarity between repeated measures $(\mathrm{CV})]$ reliability. Previous $\mathrm{CV}$ of 
Table 4 Physiological responses during arm cranking and subsequent arterial occlusion.

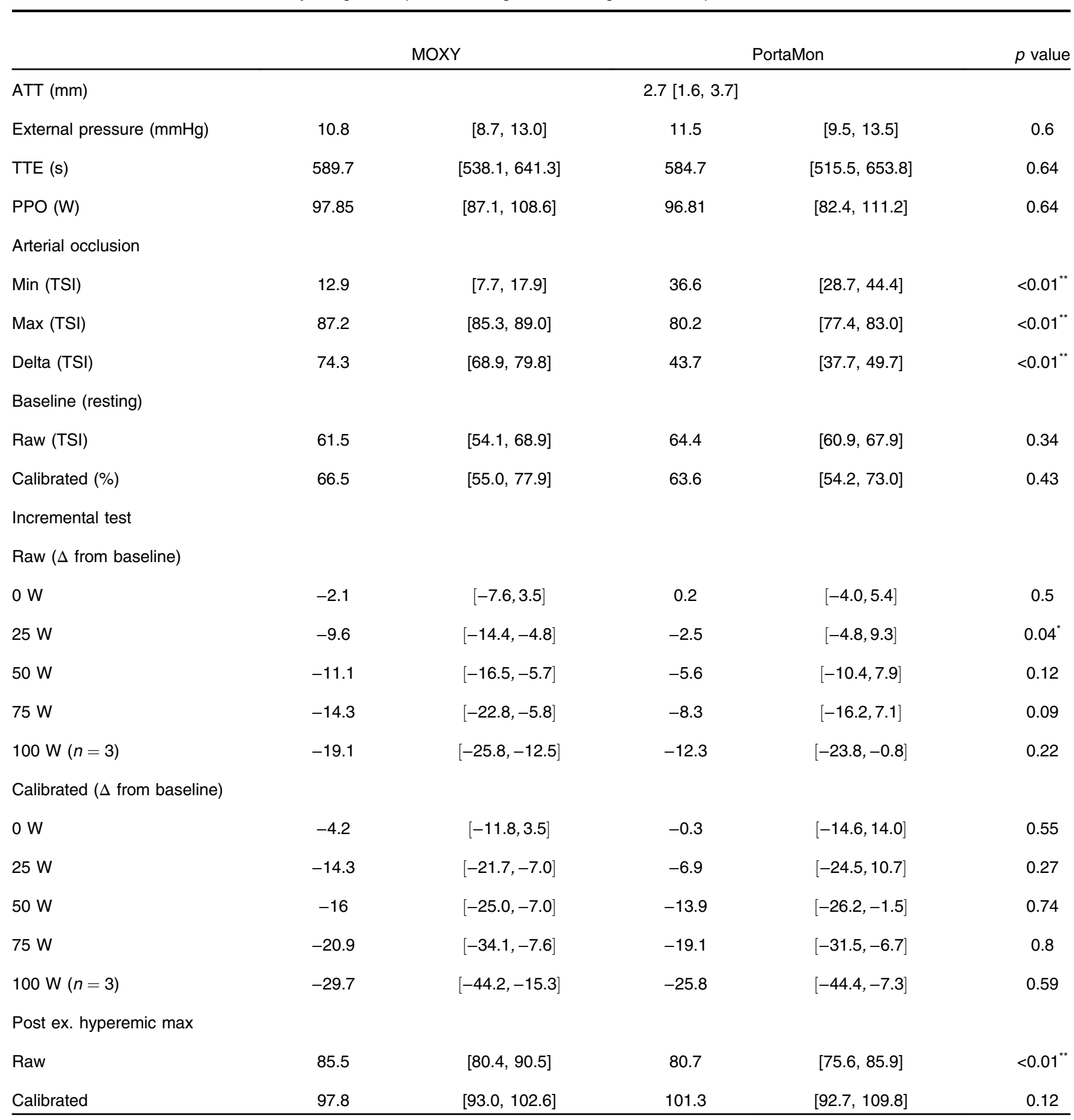

Note: ATT, adipose tissue thickness; TTE, time to exhaustion; PPO, peak power output.

${ }^{*} P=<0.05$

${ }^{* *} P=<0.01$.

various NIRS monitors report resting TSI of the vastus lateralis between $2.3 \%$ and $5.8 \% .^{25,35-37}$ These baseline TSI values are similar to that observed in the MOXY devices in our study $(5.7 \%$ to $6.2 \%$ ). Interestingly, the present $\mathrm{CV}$ for the PortaMon devices is lower than that previously reported $1.8 \%$ to $2.5 \%$ versus $4.7 \% .^{25}$ However, this variation may be due to differences in the precise anatomical location the device is placed, leg position (supine versus seated), gender (male versus male and female), and health status of the participants (healthy versus chronic heart failure).
The acceptable test-retest reliability can be partially attributed to the homogeneous group of participants used in the present study, with an adipose layer of $<7 \mathrm{~mm}$. Therefore, caution is advised if using the devices on participants with greater adipose thickness and or when taking NIRS measures from an alternative anatomical location. Furthermore, small differences in ambient and muscular temperature may influence day-today variability, ${ }^{4}$ as may slight changes in posture. ${ }^{38}$ It is likely that in the present study, controlled environmental conditions, 

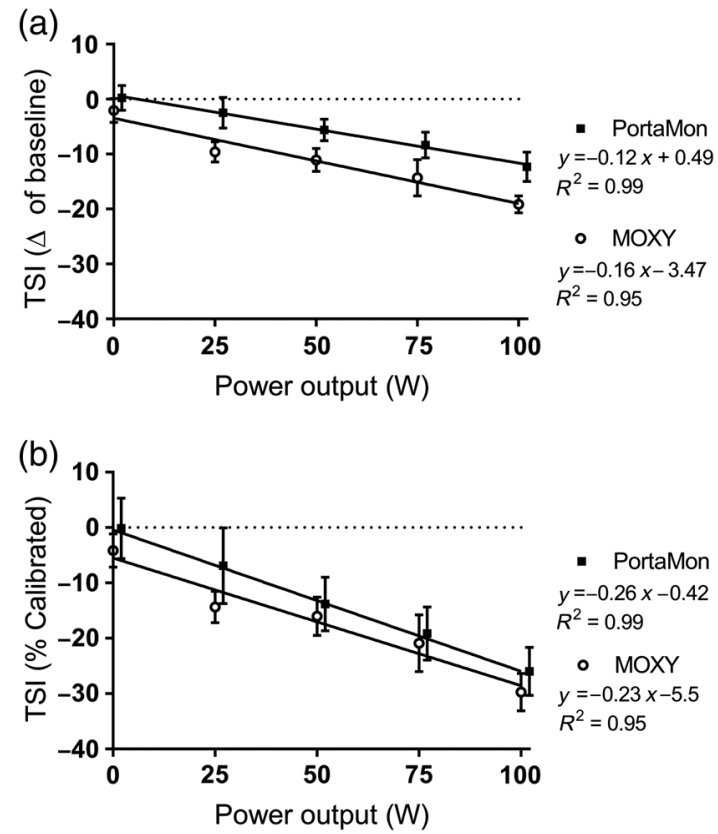

Fig. 4 (a) Relationship between power output and raw $\Delta$ TSI and (b) calibrated (\%) values during incremental arm cranking (mean \pm SEM).

(a)
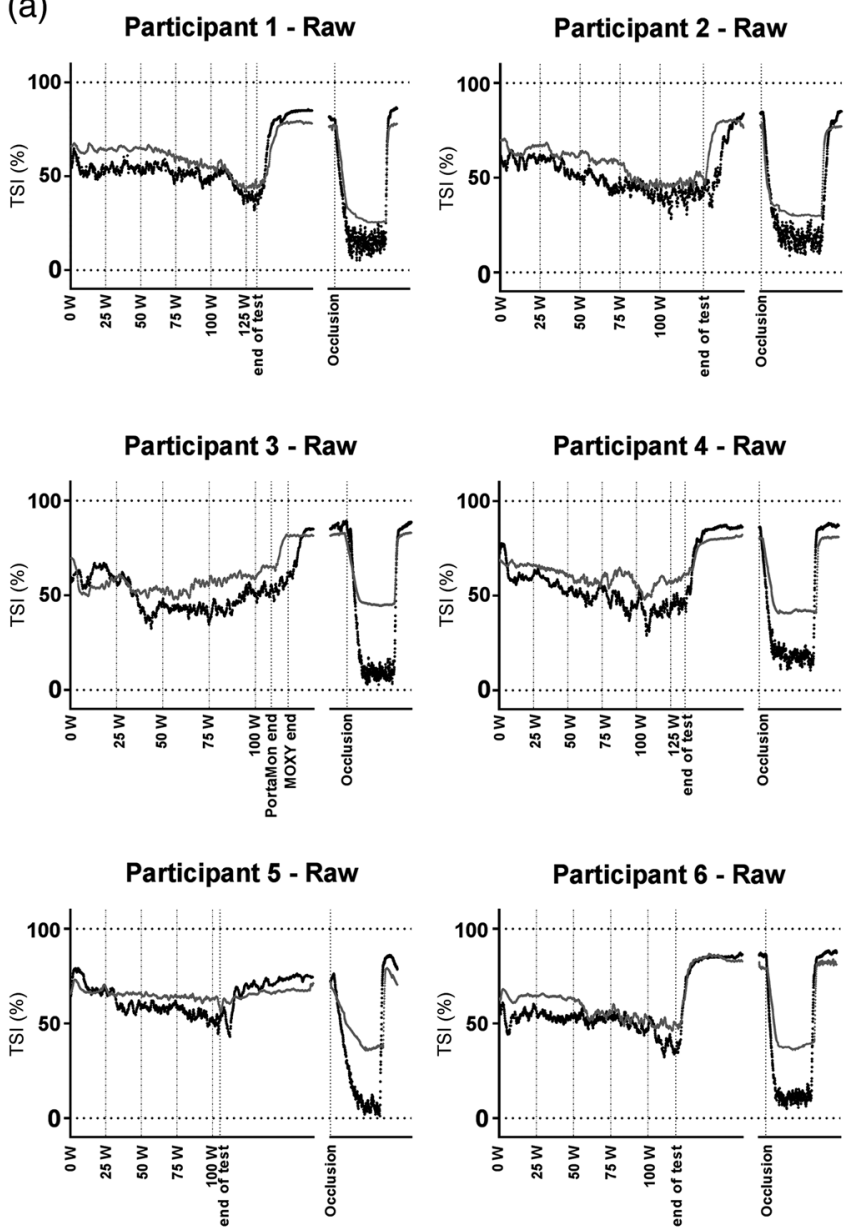

abstinence of exercise between testing days and a supine body position attenuated the variance observed, contributing toward the very strong test-retest reliability of the devices.

Resting TSI values in the brain are used as threshold values for clinical hypoxia in SR devices such as the INVOS 5100C, EQUANOX Classic 7600, and FORE-SIGHT Elite ${ }^{39}$ although this has been criticized given the differences in patient skull geometry and consequently light transport. In muscle, the InSpectra used second derivative spectroscopy to calculate a resting TSI in muscle that was suggested to report on tissue dysoxia in shock states. ${ }^{40}$ This used the thenar (base of thumb) muscle due to the very small layers of overlaying adipose tissue in this area. In muscle groups more relevant to exercise physiologists and sports scientists, resting TSI values have been reported in calf muscle in compression clothing studies ${ }^{38}$ and in the gastrocnemius during normobaric hypoxia. ${ }^{41}$ When subjected to a normobaric hypoxic environment, the mean SRSmeasured TSI change at rest, compared with the normoxic condition, was $-2.5 \%$. The authors report this finding to not statistically differ between conditions.

\subsection{Comparing Devices During Isometric Exercise}

There is no difference in $\Delta$ TSI between two models of the same device during isometric exercise. However, significant

(b)

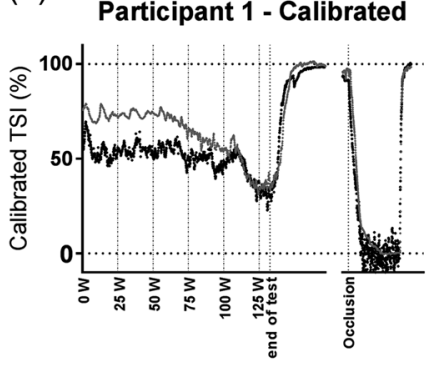

Participant 3 - Calibrated

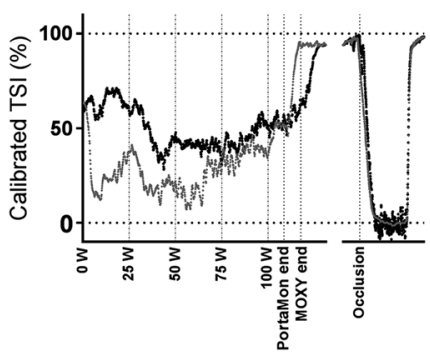

Participant 5 - Calibrated

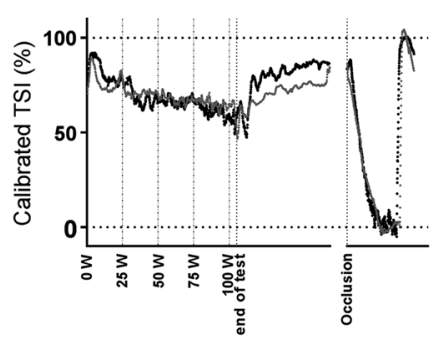

Participant 2 - Calibrated

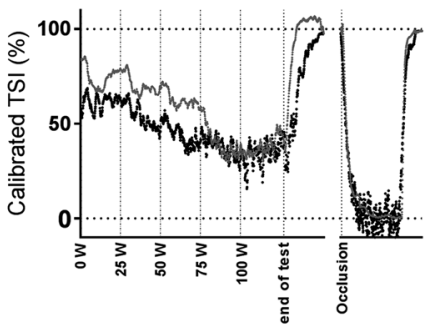

Participant 4 - Calibrated

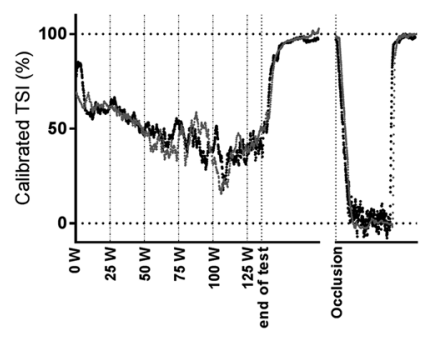

Participant 6 - Calibrated

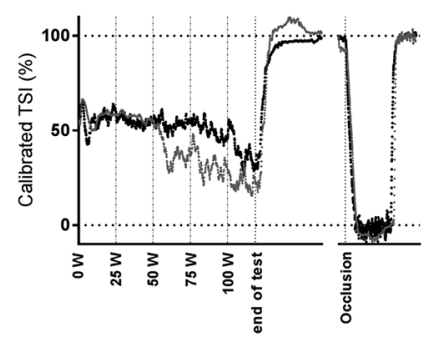

Fig. 5 (a) Individual participant TSI traces for raw and (b) calibrated values during arm cranking and arterial occlusion (MOXY = black line; PortaMon = gray line). 
differences in $\triangle \mathrm{TSI}$ are apparent when comparing MOXY versus PortaMon. The MOXY devices report a greater absolute $\Delta$ TSI change when compared with the PortaMon at both $30 \%$ and $50 \%$ MVC. The $\Delta$ TSI observed with the current PortaMon devices is similar to that of a previous study, also utilizing a PortaMon device, ${ }^{8}$ reporting a $\Delta$ TSI reduction of $\sim 6 \%$ and $11 \%$ from baseline at $30 \%$ and $50 \%$ MVC, respectively. Very few studies have utilized the MOXY device, however, of those available, the present $\Delta \mathrm{TSI}$ at $30 \% \mathrm{MVC}$ is similar to that observed in the gastrocnemius by supine individuals undertaking a plantar flexion exercise against a $6-\mathrm{kg}$ weight $(\sim 37 \%$ versus $\sim 38 \%) .{ }^{42}$

Both devices report appropriately that there is a decrease in muscle oxygenation during isometric exercise. However, unlike the situation for male subjects at rest, there are significant differences in the extent of desaturation observed during exercise. Is it possible to determine which one is likely to be "correct"? In one sense there is no single "correct" value as, given the differences in source:detector separation, the NIRS and PortaMon are reporting on different regions of the muscle (and having to correct for different amounts of surface tissue). However, some limits can be set by more invasive methods. Comparing oxygen saturation differences between femoral venous and radial arterial blood with the $\Delta$ TSI changes observed in the present study may identify whether one device is more comparable to direct methods than the other. In trained subjects, femoral venous oxygen saturations of $21 \%$ and arterial oxygen saturation of $96 \%$ were measured following maximal leg extensions. ${ }^{43}$ Assuming the NIRS signal is an approximately equal contribution of arterial, capillary, and venous saturation, one would expect the absolute muscle oxygen saturation to be no lower than $55 \%$ in this case. Even allowing for the fact that femoral venous oxygen saturation represents the sum of all blood returning from the exercising leg, whereas the NIRS signal originates in the exercising muscle only, ${ }^{44}$ the almost $60 \%$ drop in TSI in the MOXY device at only 50\% MVC seems rather high. On the other hand, as has been noted, ${ }^{45}$ the lack of a correction for the adipose layers can result in an underestimation of TSI changes using SRS methods. The variation between devices during the MVC exercise could be explained by the muscle contraction causing interference to the arterial inflow, therefore an increasing intramuscular pressure inducing ischemia. As such, the need to assess the devices during dynamic exercise provides an opportunity to understand the dynamic TSI range of the devices without the potential complication of ischemia.

\subsection{Comparing Devices During Dynamic Exercise}

During the incremental arm-cranking task, both NIRS devices demonstrated a continuous decline in TSI as power output increases until volitional fatigue. While some previous investigations have reported an early decline in muscle oxygen saturation during incremental arm exercise followed by a plateau at $\sim 50 \% \mathrm{VO}_{2 \text { max }},{ }^{46}$ the general trend in TSI of the current study (Table 4) is closer to studies where the biceps brachii TSI progressively decreases throughout arm-cranking exercise. ${ }^{5}$ Interestingly, the continuous decline in mean TSI at each stage of the test (Table 4), masked significant individual variation; in one person (participant 3) an increase in TSI was seen following the initial fall. The fact that both devices showed the same unusual trend lends confidence to the idea, that both this individual has differences in the balance between flow and metabolism as exercise proceeds and that both PortaMon and MOXY can detect this effect. By selecting participants and an anatomical location with very low ATT, and controlling external pressure at $\sim 10 \mathrm{mmHg}$, variables previously identified to influence TSI values between devices were controlled. Nevertheless, we still observed a larger dynamic range in the study in the MOXY compared to the PortaMon.

\subsection{Comparing Devices During Occlusion/Reactive Hyperemia and the Effect of Physiological Calibration}

In an attempt to further explore the remaining differences, we performed a "physiological calibration" of the two devices, comparing the dynamic exercise data from the biceps brachii to an individual subject and machine-specific $0 \%$ to $100 \%$ range determined by a nominal $0 \%$ following arterial occlusion and a nominal $100 \%$ following the reactive hyperemia after that occlusion. During the arterial occlusion (Fig. 4), both devices fell to a fixed low level below baseline within the first $3 \mathrm{~min}$ following occlusion and both showed reactive hyperemia following cuff release with the TSI rising above baseline. This contrasts with a recent study measuring TSI following 3 min arterial occlusion comparing in older adult patients $(66 \pm 9$ year $)$ scheduled for elective coronary artery bypass grafting surgery. ${ }^{22}$ In these older patients, 3 min occlusion was not enough to decrease TSI to a stable fixed baseline, probably due to a decrease in muscle oxygen consumption in this group compared to ours.

Despite choosing subjects groups with a similar baseline TSI, significant differences were seen in ischemia and reperfusion between the PortaMon and the MOXY. The range (hyperemia MAX minus occlusion MIN) in the PortaMon was $\Delta 43.7 \%$ whereas in the same subjects the MOXY change was $\Delta 74.3 \%$. However, when calibrated against the physiological range observed during and postischemia, there is now no statistically significant difference among devices during the arm-crank exercise, nor the maximum value obtained during reactive hyperemia postexercise. This suggests that both methods are equally good at measuring muscle oxygenation changes in males with low ATT.

\subsection{Comparing Algorithms}

The PortaMon uses the well-established modified BeerLambert method for measuring changes in chromophore concentrations ${ }^{47}$ and SRS for measuring absolute tissue oxygen saturation. ${ }^{20}$ The MOXY uses Beer-Lambert and a proprietary SR algorithm based on a US patent. ${ }^{48}$ Pathlength data are generated from a Monte Carlo Model, smoothed and used to generate a matrix of diffuse reflectance data at discrete values of tissue and sensor optical properties. This matrix is then used to convert the measured diffuse wavelength data to tissue optical properties, from which the oxygen saturation measure can be calculated. In their basic form, neither SRS nor SR algorithms take into account prior knowledge about individual differences in, for example, adipose layer thickness, resulting in some of the variability seen in this study. Some simple NIRS devices, such

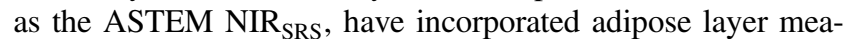
surements to improve the accuracy of their algorithms. ${ }^{49}$ In principle, both PortaMon and MOXY methods described in this paper could make similar adjustments. Previous authors have incorporated ATTs of individual subjects as measured by ultrasound combined with SRS measurements in a two layer (adipose/muscle) Monte Carlo model to directly calculate muscle 
$\mu_{\mathrm{a}}{ }^{45} \mathrm{~A}$ similar individualized measure should be possible for the MOXY device, decreasing the dependence of the TSI value on adipose layer thickness.

The MOXY device reports a significantly larger dynamic range when an arterial occlusion is applied, despite a shorter source:detector length when compared with the PortaMon. It has been suggested that the ischemic stimulus is more extensive in the muscle compared with the subdermal tissue, ${ }^{16}$ therefore, the greater dynamic range reported by the MOXY may be related to the SR method enabling a greater muscle contribution than the SRS despite the smaller source:detector separation in the MOXY. However, it is also likely in part a function of algorithm optimization and specialization. The MOXY is designed to be used on muscle and could reasonably be expected to have been optimized for this system. The PortaMon, although designed to be used on muscle, makes no concessions or alterations to the basic SRS algorithm compared to similar Artinis devices used to measure brain function.

The minimum and maximum raw TSI values reported for the PortaMon $(\Delta 43.7 \%)$ are similar to that previously reported for the NIRO $200-\mathrm{NX}^{22,50}$ following an arterial occlusion applied at the upper arm $(\Delta 33 \%)$. This finding is unsurprising given that both the NIRO 200-NX and PortaMon both use SRS methods. However, the larger dynamic range reported by the MOXY $(\Delta 74.3 \%)$ surpasses that of alternative SR devices such as the FORE-SIGHT elite $(\Delta 46 \%)$ and INVOS5100C $(\Delta 36 \%){ }^{22}$ This illustrates the difficulty in comparing different SR devices, which use different algorithms and are likely sensitive to differences in the source:detector separation even when determining basic calibration ranges. The INVOS $(3$ and $4 \mathrm{~cm}$ ) and FORESIGHT $(2.5$ and $5 \mathrm{~cm})$ use significantly larger source:detector separations than the MOXY $(1.2$ and $2.5 \mathrm{~cm})$ and, given their clinical interests, their algorithm development has likely focused more on brain than muscle measurements. However, in the absence of devices from these manufacturers with similar source:detector separations and the proprietary nature of the individual algorithms, it is impossible to speculate further at to the cause of these differences.

\subsection{Real World Applications}

The MOXY is designed to be of use to individuals in a variety of sporting environments. There have been suggestions as to how NIRS might be of use for individual athletes and coaches in, for example, tracking and optimizing training. However, in many cases, these have merely sought to mimic currently available tools, such as lactate thresholds, albeit with the benefit of being noninvasive. ${ }^{51,52}$ Even then, differences in optical properties of individuals make these measurements unreliable in many cases. ${ }^{53}$ No one has yet been able to demonstrate a specific benefit of using muscle NIRS to track training in an individual. What is required is a proper randomized trial comparing the use of NIRS to optimize training with current standard methods. The advent of relatively low-cost devices, such as the MOXY, makes such a trial more accessible. However, this work here suggests that, even if successful, the results of such a trial might remain specific for the NIR device used and not necessarily transferable to other spectrometers. This study has focused solely on the TSI values produced by the MOXY and PortaMon devices. Future studies could address the tHb values reported by the MOXY device and compare these with the (rarely reported) scaled $\mathrm{tHb}$ that can be derived from SRS methods.
Subcutaneous fat is known to have a large impact on NIRS signal. While high ATT results in less light absorption and therefore a stronger signal, the metabolically inactive fat tissue, coupled with lower hemoglobin levels will result in less signal change ${ }^{54}$ i.e., a blunted dynamic range. Given the observed influence of high ATT on resting TSI in the MOXY device and the small source:detector separation used, it is not clear how valid the current algorithm is for use in athletes with higher ATT; this includes a significant number of female athletes. In order to fully utilize the MOXY device in individuals with ATT $>7 \mathrm{~mm}$, the source-detector distance should be increased accordingly. Commercial muscle NIRS devices with larger source-detection distances are widely available. ${ }^{6}$ Future studies should measure TSI trend during dynamic exercise of those with ATT between 7 and $15 \mathrm{~mm}$ and determine whether the influence of ATT can be removed by applying an ischemic calibration as has been shown previously for muscle oxygen consumption measurements previously. ${ }^{55}$

\section{Conclusion}

This study shows that both MOXY and PortaMon devices produce acceptable TSI measures during supine rest and demonstrate a similar trend during dynamic exercise under appropriately controlled conditions in specific subject groups. During ischemia (arterial occlusion and isometric contractions), the MOXY device reports a greater raw dynamic range when compared with the PortaMon. However, by applying a physiological calibration following an arterial occlusion, the MOXY and PortaMon devices report similar changes from baseline during arm-crank exercise. Due to the limitations of calibrating NIRS, it is not possible to determine which device is a more accurate measure of absolute muscle oxygen saturation. Although, the PortaMon demonstrates a greater repeatability at rest, this may simply be reflective of the smaller dynamic range observed. The MOXY is marketed at athletes and coaches and priced accordingly $(<£ 1000)$, whereas the higher priced PortaMon $(<£ 10,000)$ tends to be used primarily by research scientists. For the researcher, the PortaMon has the advantage of measuring and reporting more NIRS parameters. At least in the case of vastus lateralis measurements, the PortaMon also appears to be less sensitive to variations in adipose layer thickness and applied external pressure. We recommend the application of the MOXY device primarily on individuals with a low ATT and that the external pressure applied to hold the device in place is either standardized upon each application or kept below $20 \mathrm{mmHg}$.

\section{Disclosures}

No conflicts of interest, financial or otherwise, are declared by the authors.

\section{Acknowledgments}

We thank the UK Engineering and Physical Sciences Research Council (EPSRC) for providing the initial funding for our work in this project (EP/F005733/1). All NIRS devices used were purchased by the University of Essex and no funding or intellectual input into this work was provided by Artinis (manufacturer of the PortaMon) or Fortiori Design LLC (manufacturer of the MOXY). We are, however, grateful to Roger Schmitz (Owner/ CEO Fortiori Design LLC) for sharing with us some details of the algorithm used to measure tissue oxygen saturation in the MOXY monitor. 


\section{References}

1. B. Grassi and V. Quaresima, "Near-infrared spectroscopy and skeletal muscle oxidative function in vivo in health and disease: a review from an exercise physiology perspective," J. Biomed. Opt. 21(9), 091313 (2016).

2. M. Muthalib et al., "Reliability of near-infrared spectroscopy for measuring biceps brachii oxygenation during sustained and repeated isometric contractions," J. Biomed. Opt. 15(1), 017008 (2010).

3. M. Ferrari, M. Muthalib, and V. Quaresima, "The use of near-infrared spectroscopy in understanding skeletal muscle physiology: recent developments," Philos. Trans. R. Soc. A 369(1955), 4577-4590 (2011).

4. M. Ferrari, L. Mottola, and V. Quaresima, "Principles, techniques, and limitations of near infrared spectroscopy," Can. J. Appl. Physiol. 29(4), 463-487 (2004).

5. S.-J. C. Lusina et al., "Muscle deoxygenation of upper-limb muscles during progressive arm-cranking exercise," Appl. Physiol., Nutr., Metab. 33(2), 231-238 (2008).

6. S. Perrey and M. Ferrari, "Muscle oximetry in sports science: a systematic review," Sports Med. 1-20 (2017).

7. B. Jones, D. K. Hamilton, and C. E. Cooper, "Muscle oxygen changes following sprint interval cycling training in elite field hockey players," PLoS One 10(3), e0120338 (2015).

8. B. Jones, M. Dat, and C. E. Cooper, "Underwater near-infrared spectroscopy measurements of muscle oxygenation: laboratory validation and preliminary observations in swimmers and triathletes," $J$. Biomed. Opt. 19(12), 127002 (2014).

9. C. M. Hesford et al., "Asymmetry of quadriceps muscle oxygenation during elite short-track speed skating," Med. Sci. Sports Exercise 44(3), 501-508 (2012).

10. D.-P. Born et al., "Near-infrared spectroscopy: more accurate than heart rate for monitoring intensity in running in hilly terrain," Int. J. Sports Physiol. Perform. 12(4), 440-447 (2017).

11. J.-L. Fellahi et al., "Dynamic evaluation of near-infrared peripheral oximetry in healthy volunteers: a comparison between INVOS and EQUANOX," J. Crit. Care 28(5), 881.e1-881.e6 (2013).

12. J.-H. Lee et al., "Comparison of two devices using near-infrared spectroscopy for the measurement of tissue oxygenation during a vascular occlusion test in healthy volunteers (INVOS ${ }^{\circledR}$ vs. InSpectra ${ }^{\mathrm{TM}}$ ), " J. Clin. Monit. Comput. 29(2), 271-278 (2015)

13. S. Hyttel-Sorensen et al., "Tissue oximetry: a comparison of mean values of regional tissue saturation, reproducibility and dynamic range of four NIRS-instruments on the human forearm," Biomed. Opt. Express 2(11), 3047-3057 (2011).

14. A. Nygren, K. Rennerfelt, and Q. Zhang, "Detection of changes in muscle oxygen saturation in the human leg: a comparison of two near-infrared spectroscopy devices," J. Clin. Monit. Comput. 28(1), 57-62 (2014).

15. S. Engbers et al., "A comparison of tissue oxygen saturation measurements by 2 different near-infrared spectroscopy monitors in 21 healthy dogs," J. Vet. Emerg. Crit. Care 24(5), 536-544 (2014).

16. R. Bezemer et al., "Assessment of tissue oxygen saturation during a vascular occlusion test using near-infrared spectroscopy: the role of probe spacing and measurement site studied in healthy volunteers," Crit. Care 13(5), S4 (2009).

17. H. Gómez et al., "Characterization of tissue oxygen saturation and the vascular occlusion test: influence of measurement sites, probe sizes and deflation thresholds," Criti. Care 13(5), S3 (2009).

18. D. E. Myers et al., "Noninvasive method for measuring local hemoglobin oxygen saturation in tissue using wide gap second derivative nearinfrared spectroscopy," J. Biomed. Opt. 10(3), 034017 (2005).

19. S. Matcher and C. Cooper, "Absolute quantification of deoxyhaemoglobin concentration in tissue near infrared spectroscopy," Phys. Med. Biol. 39(8), 1295-1312 (1994).

20. S. Suzuki et al., "Tissue oxygenation monitor using NIR spatially resolved spectroscopy," Proc. SPIE 3597, 582-592 (1999).

21. T. Muellner et al., "New instrument that uses near-infrared spectroscopy for the monitoring of human muscle oxygenation," J. Trauma Acute Care Surg. 46(6), 1082-1084 (1999).

22. K. Steenhaut et al., "Evaluation of different near-infrared spectroscopy technologies for assessment of tissue oxygen saturation during a vascular occlusion test," J. Clin. Monit. Comput. 31(6), 1151-1158 (2017).
23. M. Thavasothy et al., "A comparison of cerebral oxygenation as measured by the NIRO 300 and the INVOS 5100 near-infrared spectrophotometers," Anaesthesia 57(10), 999-1006 (2002).

24. K. Cornachione, J. McLaren, and D. P. Heil, "Use of a wireless NIRS monitor to track changes in muscle oxygenation for laboratory-based Nordic skiing test protocol," in Science and Skiing VI, E. Müller et al., Eds., pp. 369-376, Meyer \& Meyer Sport (UK) Ltd., Maidenhead (2014).

25. V. M. Niemeijer et al., "Test-retest reliability of skeletal muscle oxygenation measurements during submaximal cycling exercise in patients with chronic heart failure," Clin. Physiol. Funct. Imaging 37(1), 68-78 (2017)

26. B. R. Scott et al., "Reliability of telemetric electromyography and nearinfrared spectroscopy during high-intensity resistance exercise," $J$. Electromyogr. Kinesiol. 24(5), 722-730 (2014).

27. H. Gómez et al., "Use of non-invasive NIRS during a vascular occlusion test to assess dynamic tissue $\mathrm{O}_{2}$ saturation response," Intensive Care Med. 34(9), 1600-1607 (2008).

28. W. Hopkins et al., "Progressive statistics for studies in sports medicine and exercise science," Med. Sci. Sports Exercise 41(1), 3-13 (2009).

29. G. Atkinson and A. M. Nevill, "Statistical methods for assessing measurement error (reliability) in variables relevant to sports medicine," Sports Med. 26(4), 217-238 (1998).

30. F. Faul et al., "G* power 3: a flexible statistical power analysis program for the social, behavioral, and biomedical sciences," Behav. Res. Methods 39(2), 175-191 (2007).

31. Y. N. Bhambhani, "Muscle oxygenation trends during dynamic exercise measured by near infrared spectroscopy," Can. J. Appl. Physiol. 29(4), 504-523 (2004)

32. S. Homma, T. Fukunaga, and A. Kagaya, "Influence of adipose tissue thickness on near infrared spectroscopic signals in the measurement of human muscle," J. Biomed. Opt. 1(4), 418-424 (1996).

33. C. E. Cooper et al., "Comparison of local adipose tissue content and SRSderived NIRS muscle oxygenation measurements in 90 individuals," in Oxygen Transport to Tissue XXXI, pp. 177-181, Springer (2010).

34. A. D. B. Chant, "The effects of posture, exercise, and bandage pressure on the clearance of 24na from the subcutaneous tissues of the foot," $B r$. J. Surg. 59(7), 552-555 (1972).

35. M. Ihsan et al., "Muscle oxygenation and blood volume reliability during continuous and intermittent running," Int. J. Sports Med. 34(07), 637-645 (2013)

36. L. A. Roberts et al., "Effects of cold water immersion and active recovery on hemodynamics and recovery of muscle strength following resistance exercise," Am. J. Physiol.-Regul., Integr. Comp. Physiol. 309(4), R389-R398 (2015).

37. C. Thiel et al., "Reproducibility of muscle oxygen saturation," Int. J. Sports Med. 32(04), 277-280 (2011).

38. A. Bringard et al., "Effects of compression tights on calf muscle oxygenation and venous pooling during quiet resting in supine and standing positions," J. Sports Med. Phys. Fitness 46(4), 548 (2006).

39. A. Deschamps et al., "Cerebral oximetry monitoring to maintain normal cerebral oxygen saturation during high-risk cardiac surgery: a randomized controlled feasibility trial," J. Am. Soc. Anesthesiol. 124(4), 826836 (2016)

40. A. Lima et al., "Low tissue oxygen saturation at the end of early goaldirected therapy is associated with worse outcome in critically ill patients," Crit. Care 13(5), S13 (2009).

41. T. Rupp and S. Perrey, "Effect of severe hypoxia on prefrontal cortex and muscle oxygenation responses at rest and during exhaustive exercise," in Oxygen Transport to Tissue XXX, pp. 329-334, Springer (2009).

42. M. D. Muller et al., "Muscle oxygenation during dynamic plantar flexion exercise: combining BOLD MRI with traditional physiological measurements," Physiol. Rep. 4(20), e13004 (2016).

43. M. Mourtzakis et al., "Hemodynamics and $\mathrm{O}_{2}$ uptake during maximal knee extensor exercise in untrained and trained human quadriceps muscle: effects of hyperoxia," J. Appl. Physiol. 97(5), 1796-1802 (2004)

44. M. J. MacDonald et al., "Comparison of femoral blood gases and muscle near-infrared spectroscopy at exercise onset in humans," $J$. Appl. Physiol. 86(2), 687-693 (1999).

45. D. Geraskin et al., "Algorithms for muscle oxygenation monitoring corrected for adipose tissue thickness," in Advances in Medical Engineering, pp. 384-388 (2007) 
46. S. Muraki, N. Tsunawake, and M. Yamasaki, "Limitation of muscle deoxygenation in the triceps during incremental arm cranking in women," Eur. J. Appl. Physiol. 91(2-3), 246-252 (2004).

47. D. Delpy and M. Cope, "Quantification in tissue near-infrared spectroscopy,” Philos. Trans. R. Soc. London B: Biol. Sci. 352(1354), 649-659 (1997).

48. R. Schmitz, "Systems and methods for measuring oxygenation," U.S. Patent No. 8, 941, 830, Washington, D.C. (2015).

49. S. Takagi et al., "Aerobic training enhances muscle deoxygenation in early post-myocardial infarction," Eur. J. Appl. Physiol. 116(4), 673685 (2016).

50. K. Steenhaut et al., "Evaluation of different near-infrared spectroscopy technologies for assessment of tissue oxygen saturation during a vascular occlusion test," J. Clin. Monit. Comput. 31(6), 1151-1158 (2017).

51. Y. N. Bhambhani, S. M. Buckley, and T. Susaki, "Detection of ventilatory threshold using near infrared spectroscopy in men and women,' Med. Sci. Sports Exercise 29(3), 402-409 (1997).
52. R. Belardinelli et al., "Changes in skeletal muscle oxygenation during incremental exercise measured with near infrared spectroscopy," Eur. J. Appl. Physiol. Occup. Physiol. 70(6), 487-492 (1995).

53. C. Angus et al., "Estimation of lactate threshold by near infrared spectroscopy," Adv. Exp. Med. Biol. 471, 283-288 (1998).

54. K. K. McCully and T. Hamaoka, "Near-infrared spectroscopy: what can it tell us about oxygen saturation in skeletal muscle?" Exercise Sport Sci. Rev. 28(3), 123-127 (2000).

55. T. E. Ryan et al., "Noninvasive evaluation of skeletal muscle mitochondrial capacity with near-infrared spectroscopy: correcting for blood volume changes," J. Appl. Physiol. 113(2), 175-183 (2012).

Biographies for the authors are not available. 